\title{
ON THE ROLE OF NUMERICAL VISCOSITY IN THE STUDY OF THE LOCAL LIMIT OF NONLOCAL CONSERVATION LAWS
}

\author{
Maria Colombo ${ }^{1}$, Gianluca Crippa ${ }^{2, *}$, Marie GrafF $^{3}$ and Laura V. Spinolo ${ }^{4}$
}

\begin{abstract}
We deal with the numerical investigation of the local limit of nonlocal conservation laws. Previous numerical experiments seem to suggest that the solutions of the nonlocal problems converge to the entropy admissible solution of the conservation law in the singular local limit. However, recent analytical results state that (i) in general convergence does not hold because one can exhibit counterexamples; (ii) convergence can be recovered provided viscosity is added to both the local and the nonlocal equations. Motivated by these analytical results, we investigate the role of numerical viscosity in the numerical study of the local limit of nonlocal conservation laws. In particular, we show that Lax-Friedrichs type schemes may provide the wrong intuition and erroneously suggest that the solutions of the nonlocal problems converge to the entropy admissible solution of the conservation law in cases where this is ruled out by analytical results. We also test Godunov type schemes, less affected by numerical viscosity, and show that in some cases they provide an intuition more in accordance with the analytical results.
\end{abstract}

Mathematics Subject Classification. 35L65, 65M12.

Received February 19, 2019. Accepted October 29, 2021.

\section{INTRODUCTION}

\subsection{Theoretical framework}

We consider nonlocal conservation laws in the form

$$
\partial_{t} \rho+\partial_{x}[\rho b(\rho * \eta)]=0,
$$

where the unknown is $\rho:[0,+\infty) \times \mathbb{R} \rightarrow \mathbb{R}, b: \mathbb{R} \rightarrow \mathbb{R}$ is a given Lipschitz continuous function and $\eta: \mathbb{R} \rightarrow \mathbb{R}$ is a smooth convolution kernel satisfying

$$
\eta \in C_{c}^{\infty}(\mathbb{R}), \quad \eta(x)=0 \quad \text { if }|x| \geq 1, \quad \eta \geq 0, \quad \int_{\mathbb{R}} \eta(x) \mathrm{d} x=1 .
$$

Keywords and phrases. Numerical viscosity, nonlocal conservation law, nonlocal traffic models, nonlocal-to-local limit.

1 EPFL SB, Station 8, CH-1015 Lausanne, Switzerland.

2 Departement Mathematik und Informatik, Universität Basel, Spiegelgasse 1, CH-4051 Basel, Switzerland.

3 Department of Mathematics, University of Auckland, Private Bag 92019, Auckland 1142, New Zealand.

4 IMATI-CNR, Via Ferrata 5, I-27100 Pavia, Italy.

*Corresponding author: gianluca.crippa@unibas.ch 
In recent years, nonlocal conservation laws have been used to model, among others, sedimentation [3], pedestrian $[10]$ and vehicular $[4,7]$ traffic. In particular, in the case of traffic models $\rho$ represents the density of agents (cars, pedestrians) and $b$ their speed. The convolution term models the fact that drivers and pedestrians decide their velocity based on the density of agents around them. Loosely speaking, the radius of the support of $\eta$ represents the visual range of drivers and pedestrians. Existence and uniqueness results for the Cauchy problem obtained by coupling (1.1) with an initial datum have been obtained in several works, see for instance $[4,10,13]$.

In this work we deal with the numerical investigation of the local limit. More precisely, we consider a parameter $\varepsilon>0$ and we rescale $\eta$ by setting $\eta_{\varepsilon}(x):=\varepsilon^{-1} \eta(x / \varepsilon)$, in such a way that, when $\varepsilon \rightarrow 0^{+}, \eta_{\varepsilon}$ converges weakly-* in the sense of measures to the Dirac delta. We fix an initial datum $\bar{\rho}: \mathbb{R} \rightarrow \mathbb{R}$ and we consider the family of Cauchy problems

$$
\left\{\begin{array}{l}
\partial_{t} \rho_{\varepsilon}+\partial_{x}\left[\rho_{\varepsilon} b\left(\rho_{\varepsilon} * \eta_{\varepsilon}\right)\right]=0 \\
\rho_{\varepsilon}(0, x)=\bar{\rho}(x)
\end{array}\right.
$$

When $\varepsilon \rightarrow 0^{+}$(i.e. in the local limit), the above Cauchy problem formally boils down to the conservation law

$$
\left\{\begin{array}{l}
\partial_{t} \rho+\partial_{x}[\rho b(\rho)]=0 \\
\rho(0, x)=\bar{\rho}(x)
\end{array}\right.
$$

The by now classical theory by Kružkov [17] states that, if $\bar{\rho} \in L^{\infty}(\mathbb{R})$, the above problem has a unique entropy admissible solution, i.e. loosely speaking a unique distributional solution that is consistent with the Second Principle of Thermodynamics. Motivated by numerical experiments obtained with a Lax-Friedrichs type scheme, in [2] P. Amorim, R. Colombo and A. Teixeira posed the following question.

Question 1. Can we rigorously justify the local limit? Namely, does the solution $\rho_{\varepsilon}$ of (1.2) converge to the entropy admissible solution $\rho$ of (1.3) as $\varepsilon \rightarrow 0^{+}$, in some suitable topology?

In [2] the authors provide numerical evidence supporting a positive answer to Question 1 . See also $[1,4,14,15]$. In the special case where the solution of (1.3) is regular, and the convolution kernel is an even function, Zumbrun [22] showed that the solutions of (1.2) converge to the entropy admissible solution of (1.3) as $\varepsilon \rightarrow 0^{+}$. Despite this positive result, the answer to Question 1 is actually negative in general. More precisely, in [11] we exhibit some analytical counterexamples that rule out the convergence of the solutions of (1.2) to the entropy admissible solution of (1.3) (see Sect. 3 for an overview of these counterexamples). Very loosely speaking, one of the main goals of the present paper is to provide insights on the reasons why the numerical evidence in [2] provides the wrong intuition on the nonlocal-to-local limit from (1.2) to (1.3).

In [11] we also consider the "viscous counterpart" of Question 1. More precisely, we fix a viscosity parameter $\nu>0$ and add a viscous second order term to the right hand side of both (1.2) and (1.3). We arrive at

$$
\left\{\begin{array}{l}
\partial_{t} \rho_{\varepsilon \nu}+\partial_{x}\left[\rho_{\varepsilon \nu} b\left(\rho_{\varepsilon \nu} * \eta_{\varepsilon}\right)\right]=\nu \partial_{x x}^{2} \rho_{\varepsilon \nu} \\
\rho_{\varepsilon \nu}(0, x)=\bar{\rho}(x)
\end{array}\right.
$$

and

$$
\left\{\begin{array}{l}
\partial_{t} \rho_{\nu}+\partial_{x}\left[\rho_{\nu} b\left(\rho_{\nu}\right)\right]=\nu \partial_{x x}^{2} \rho_{\nu} \\
\rho_{\nu}(0, x)=\bar{\rho}(x)
\end{array}\right.
$$

respectively. This yields the "viscous counterpart" of Question 1, namely

Question 2. Fix $\nu>0$. Does the solution $\rho_{\varepsilon \nu}$ of (1.4) converge to the solution $\rho_{\nu}$ of (1.5), when $\varepsilon \rightarrow 0^{+}$?

The answer to Question 2 is largely positive. More precisely, Theorem 1.1 of [11] states in particular that, for every $\nu>0$ and $T>0$, the family $\rho_{\varepsilon \nu}$ converges to $\rho_{\nu}$ in the strong topology of $L^{2}([0, T] \times \mathbb{R})^{1}$. See also [9]. To

\footnotetext{
${ }^{1}$ The precise results collected in Theorem 1.1 of [11] are actually stronger and in particular apply to the case of several space dimensions.
} 
conclude the overview of the analytical results, we quote Proposition 1.2 of [11], which establishes the "nonlocal" vanishing viscosity limit $\nu \rightarrow 0^{+}$from (1.4) to (1.2), whereas the "local" vanishing viscosity limit from (1.5) to (1.3) is a classical result by Kružkov [17]. Summing up, we have the following convergence scheme:

$$
\begin{aligned}
& \partial_{t} \rho_{\varepsilon \nu}+\partial_{x}\left[\rho_{\varepsilon \nu} b\left(\rho_{\varepsilon \nu} * \eta_{\varepsilon}\right)\right]=\nu \partial_{x x}^{2} \rho_{\varepsilon \nu} \frac{\varepsilon \rightarrow 0^{+}}{([11], \text { Thm. 1.1) }} \partial_{t} \rho_{\nu}+\partial_{x}\left[\rho_{\nu} b\left(\rho_{\nu}\right)\right]=\nu \partial_{x x}^{2} \rho_{\nu} \\
& \nu \rightarrow 0^{+} \downarrow\left([11], \text { Prop. 1.2) } \quad \nu \rightarrow 0^{+} \downarrow\right. \text { Kružkov's Theorem } \\
& \partial_{t} \rho_{\varepsilon}+\partial_{x}\left[\rho_{\varepsilon} b\left(\rho_{\varepsilon} * \eta_{\varepsilon}\right)\right]=0 \quad \underset{\varepsilon \rightarrow 0^{+}}{\stackrel{\text { False in general }}{\longrightarrow}} \quad \partial_{t} \rho+\partial_{x}[\rho b(\rho)]=0 .
\end{aligned}
$$

\subsection{Numerical results}

As mentioned before, Question 1 was first raised, to the best of our knowledge, in [2]. As it is very often the case in applied mathematics, the authors of [2] discussed Question 1 by running some numerical tests, which supported a positive answer to Question 1. The answer suggested by the numerical evidence was later contradicted by the analytical results established in [11], which show that the correct answer to Question 1 is actually negative. The present paper aims at providing insights on the possible reason why the numerical evidence may suggest the wrong intuition. More precisely, the main goal of the present paper is to raise a warning flag: the numerical investigation of Question 1 is fairly subtle and numerical experiments, expecially those performed on coarse meshes, can easily provide misleading information concerning the nonlocal-to-local limit. In particular, in the present paper we investigate the role of numerical viscosity as one of the main factors that may jeopardize the reliability of the numerical experiments.

To illustrate the heart of the matter, we first point out that the numerical results in [2] are obtained by Lax-Friedrichs type schemes, which are known to have high numerical viscosity, see [20,21]. We refer to [18] for a more extended discussion, but, very loosely speaking, the numerical viscosity is a collection of finite difference terms that is the "numerical counterpart" of a viscous second order term like the one at the right hand side of the equations in (1.4) and (1.5). In other words, the presence of the numerical viscosity implies that the model equation (that is, very loosely speaking, the equation that provides the best approximation of the numerical scheme) for the Lax-Friedrichs scheme applied to the conservation law at the first line of (1.3) is actually the equation at the first line of (1.5), where the coefficient $\nu$ is of the same order as the space mesh. Similarly, when the Lax-Friedrichs scheme is applied to the nonlocal conservation law at the first line of (1.2) the model equation is actually the equation at the first line of (1.4).

We can now go back to the fact that the numerical evidence does not agree with the analytical results: a possible explanation is the following. Because of the numerical viscosity, the numerical tests in [2] may be actually capturing the convergence of $\rho_{\varepsilon \nu}$ to $\rho_{\nu}$, which holds true by Theorem 1.1 of [11]. In other words: the numerical tests were designed to suggest an answer to Question 1, but as a matter of fact, owing to the numerical viscosity, for suitable values of the parameter $\varepsilon$ and of the mesh size they suggest an answer to Question 2 . Since the two questions have opposite answers, the numerical tests may provide the wrong intuition concerning Question 1.

In the present paper we exhibit numerical experiments supporting the previous argument. In particular, we show that the numerical viscosity can jeopardize the reliability of standard numerical schemes for the study of the nonlocal-to-local limit from (1.2) to (1.3). In particular, in Sections 5.2-5.4 we consider the counterexamples in [11] showing that the answer to Question 1 is negative and we test them with the Lax-Friedrichs type scheme. For several values of the parameter $\varepsilon$ and of the mesh size the numerical results we obtain suggest that the answer to Question 1 is positive and hence provide the wrong intuition.

We remark in passing that for the sake of accuracy in the paper we run fairly time-consuming numerical experiments that go beyond the computational capacity of common laptops and require the use of more powerful servers. This allows us to test a fairly large set of parameters $\varepsilon$. Our investigation, however, was originally motivated by the goal of understanding why the numerical evidence provides in some cases the wrong intuition on the nonlocal-to-local limit: note that in the original paper [2] only one value of the mesh size and four values 
of the parameter $\varepsilon$ were tested. Note furthermore that in applied mathematics quite fast numerical experiments are often run in the attempt at gaining some insights on analytical questions. In particular, the fairly extended numerical experiments we run should not conceal the fact that the take-home message of our paper is in a nutshell that, for some values of the parameter $\varepsilon$ and of the mesh size, the numerical experiments may provide the wrong intuition.

In this work we also further investigate the role of numerical viscosity by comparing the Lax-Friedrichs type scheme with a Godunov type scheme. Lax-Friedrichs type schemes are known to have higher numerical viscosity than Godunov type schemes (see the analysis in [20,21] and Sect. 2.2 for a brief overview). Consistently, we find that in some cases the numerical results obtained with the Godunov type scheme are more in accordance with the analytical results than those obtained with the Lax-Friedrichs type scheme, see Sections 5.3 and 5.4.

Finally, we provide further insights on the relation between numerical viscosity and nonlocal-to-local limit by varying the relation between the convolution parameter $\varepsilon$ and the numerical viscosity. Since the numerical viscosity depends monotonically on the space mesh, it suffices to vary the relation between the convolution parameter $\varepsilon$ and the space mesh $h$. The numerical results obtained when $h$ is of the order of $\varepsilon^{2}$ are in some cases more in accordance with the analytical results than those obtained when $h$ is of the order of $\varepsilon$, see Section 5.4. This is again an indication of the fact that the numerical viscosity may compromise the ability of the numerical scheme to provide reliable information on the nonlocal-to-local limit. Indeed, when the numerical viscosity decays faster to 0 the numerical results are more in accordance with the analytical results. In general, the results we have obtained that are the most in accordance with the analytical results use a Godunov type scheme and a space mesh $h$ of the order of $\varepsilon^{2}$, and this again confirms that the smaller the numerical viscosity, the more reliable the numerical results. Note, however, that Godunov type schemes are still not completely satisfactory for the investigation of the nonlocal-to-local limit and that finding reliable numerical schemes to investigate the nonlocal-to-local limit is an interesting open problem, see also Section 6 for further comments on this issue.

The paper is organized as follows. In Section 2 we discuss the numerical schemes used in the present work, i.e. the Lax-Friedrichs and the Godunov schemes. In Section 3 we introduce the examples we will use in the numerical tests and we overview their main analytical properties. In Section 4 we validate our schemes by computing the numerical solutions in examples where the analytical solution is known, and by showing that the two are close. In Section 5 we introduce our main numerical results concerning the nonlocal-to-local limit. In Section 6 we draw our conclusions and we outline some possible future work. To simplify the exposition, in the paper we always focus on the case where the conservation law at the first line of (1.3) is the scalar Burgers' equation

$$
\partial_{t} \rho+\partial_{x}\left(\rho^{2}\right)=0
$$

and hence the nonlocal equation at the first line of $(1.2)$ is

$$
\partial_{t} \rho_{\varepsilon}+\partial_{x}\left(\rho_{\varepsilon}\left(\rho_{\varepsilon} * \eta_{\varepsilon}\right)\right)=0
$$

\section{Two Numerichl SCHEMES FOR THE Burgers' EQUATION}

We now discuss two numerical schemes for both the local (1.6) and nonlocal Burgers' equation (1.7). We refer to the book by LeVeque [18] for an extended discussion on numerical schemes for conservation laws.

We discretize the $(t, x)$-plane by choosing the space mesh width $h$ and the time step $\Delta t$ and by introducing the mesh points $\left(t_{n}, x_{j}\right)$ given by $x_{j}=j h, j \in \mathbb{Z}$, and $t_{n}=n \Delta t, n=0, \ldots, N, N=[T / \Delta t]+1$, where $T$ is the final time and [.] denotes the integer part. In the following we always consider a uniform mesh where $\Delta t / h=1 / 6$, which is consistent with the CFL condition. For technical reasons we also define

$$
x_{j+1 / 2}=x_{j}+h / 2=(j+1 / 2) h .
$$

The numerical schemes aim at defining a piecewise constant approximate solution $\rho_{h}$. As a matter of fact, in the following we will only define the discrete values $\rho_{j}^{n}$. The pointwise values of the approximate solutions are 
recovered by setting $\rho_{h}(t, x):=\rho_{j}^{n}$ if $\left.(t, x) \in\right] t_{n}, t_{n+1}[\times] x_{j-1 / 2}, x_{j+1 / 2}[$. We construct the approximate initial datum by setting

$$
\rho_{j}^{0}:=\frac{1}{h} \int_{x_{j-1 / 2}}^{x_{j+1 / 2}} \bar{\rho}(x) \mathrm{d} x
$$

Both the Lax-Friedrichs and the Godunov scheme are conservative methods that can be written in the form

$$
\rho_{j}^{n+1}=\rho_{j}^{n}-\frac{\Delta t}{h}\left[F_{j+1 / 2}^{n}-F_{j-1 / 2}^{n}\right],
$$

where $F_{j \pm 1 / 2}^{n}$ is the so-called numerical flux function. The two methods differ in the way one defines the value of $F_{j \pm 1 / 2}^{n}$. We now separately describe them.

\subsection{The Lax-Friedrichs method}

The Lax-Friedrichs scheme was originally designed for the nonlinear conservation law

$$
\partial_{t} \rho+\partial_{x} f(\rho)=0
$$

and it is defined by plugging into (2.2) the following numerical flux function:

$$
F_{j+1 / 2}^{n}=\frac{h}{2 \Delta t}\left(\rho_{j}^{n}-\rho_{j+1}^{n}\right)+\frac{1}{2}\left(f\left(\rho_{j}^{n}\right)+f\left(\rho_{j+1}^{n}\right)\right) .
$$

In the case of the Burgers' equation (1.6), the above expression boils down to

$$
F_{j+1 / 2}^{n}=\frac{h}{2 \Delta t}\left(\rho_{j}^{n}-\rho_{j+1}^{n}\right)+\frac{1}{2}\left(\left(\rho_{j}^{n}\right)^{2}+\left(\rho_{j+1}^{n}\right)^{2}\right) \Longrightarrow \rho_{j}^{n+1}=\frac{1}{2}\left(\rho_{j+1}^{n}+\rho_{j-1}^{n}\right)-\frac{\Delta t}{2 h}\left[\left(\rho_{j+1}^{n}\right)^{2}-\left(\rho_{j-1}^{n}\right)^{2}\right] .
$$

The numerical viscosity $\nu_{\mathrm{LF}}$ of the Lax-Friedrichs scheme satisfies $\nu_{\mathrm{LF}}=h^{2} / 2 \Delta t$, and owing to the CFL condition $\Delta t / h=1 / 6$ we get $\nu_{\mathrm{LF}}=3 h$. In other words, the numerical viscosity is of the same order as the space mesh.

Lax-Friedrichs type schemes for nonlocal conservation laws were considered in various works, see for instance in $[2-4]$. In the case of the nonlocal Burgers' equation (1.7), the numerical flux function is defined by setting

$$
F_{j+1 / 2}^{n}=\frac{h}{2 \Delta t}\left(\rho_{j}^{n}-\rho_{j+1}^{n}\right)+\frac{1}{2}\left(\rho_{j}^{n} c_{j}^{n}+\rho_{j+1}^{n} c_{j+1}^{n}\right),
$$

where $c_{j}^{n}$ is the approximate value of the convolution kernel and in the present work it is computed by the quadrature formula

$$
c_{j}^{n}=\sum_{k=-\ell}^{\ell-1} \gamma_{k} \rho_{j-k}^{n}, \quad \text { where } \quad \gamma_{k}=\int_{k h}^{(k+1) h} \eta_{\varepsilon}(y) \mathrm{d} y \quad \text { and } \quad \ell=\left[\frac{\varepsilon}{h}\right]+1 .
$$

We recall that the support of the convolution kernel $\eta_{\varepsilon}$ is always contained in the interval $[-\varepsilon, \varepsilon]$ and we remark that the above formula provides a reasonable approximation of a convolution only when the parameter $\varepsilon$ is sufficiently large compared to the space mesh $h$. By plugging (2.4) into (2.2) we arrive at

$$
\rho_{j}^{n+1}=\frac{1}{2}\left(\rho_{j+1}^{n}+\rho_{j-1}^{n}\right)-\frac{\Delta t}{2 h}\left(\rho_{j+1}^{n} c_{j+1}^{n}-\rho_{j-1}^{n} c_{j-1}^{n}\right) .
$$




\subsection{The Godunov method}

The basic idea underpinning the Godunov scheme is to solve Riemann problems on each cell of the computational mesh. More precisely, the Godunov scheme for the nonlinear conservation law (2.3) is obtained by plugging the numerical flux function

$$
F_{j+1 / 2}^{n}:=f\left(\rho^{*}\left(\rho_{j}^{n}, \rho_{j+1}^{n}\right)\right)
$$

into (2.2). In the previous expression, $\rho^{*}\left(\rho_{j}^{n}, \rho_{j+1}^{n}\right)$ is the value at the line $x=0$ of the entropy admissible solution of the Riemann problem between $\rho_{j}^{n}$ (on the left) and $\rho_{j+1}^{n}$ (on the right). Note that, owing to the Rankine-Hugoniot conditions, even if the solution of the Riemann problem has a discontinuity at $x=0$, the function $f(\rho)$ is continuous at $x=0$ and hence the value $f\left(\rho^{*}\right)$ is well-defined. As a matter of fact, if the flux function $f$ is convex we have the equality

$$
f\left(\rho^{*}\left(\rho_{j}^{n}, \rho_{j+1}^{n}\right)\right)= \begin{cases}\min _{\rho \in\left[\rho_{j}^{n}, \rho_{j+1}^{n}\right]} f(\rho) & \rho_{j}^{n} \leq \rho_{j+1}^{n} \\ \max _{\rho \in\left[\rho_{j+1}^{n}, \rho_{j}^{n}\right]} f(\rho) & \rho_{j}^{n} \geq \rho_{j+1}^{n},\end{cases}
$$

which in the case of the scalar Burgers' equation (1.6) implies

$$
\left(\rho^{*}\left(\rho_{j}^{n}, \rho_{j+1}^{n}\right)\right)^{2}= \begin{cases}\min _{\rho \in\left[\rho_{j}^{n}, \rho_{j+1}^{n}\right]} \rho^{2} & \rho_{j}^{n} \leq \rho_{j+1}^{n} \\ \max _{\rho \in\left[\rho_{j+1}^{n}, \rho_{j}^{n}\right]} \rho^{2} & \rho_{j}^{n} \geq \rho_{j+1}^{n} .\end{cases}
$$

Note furthermore that the Godunov scheme is known to have less numerical viscosity than the Lax-Friedrichs scheme, see [20,21]. More precisely, Tadmor in [20,21] focuses on conservative, finite difference schemes for scalar conservation laws and investigates the relation between entropy inequalities and numerical viscosity. At the analytical level, the relation is very well understood: the strong limit $\nu \rightarrow 0^{+}$of the vanishing vanishing viscosity approximation (1.5) is an entropy admissible solution of the conservation law (1.3). At the numerical level, Tadmor in [20,21] discusses the class of so-called E-schemes, which was introduced in [19] and is the class of schemes that are entropy stable with respect to all convex entropies. The Lax-Friedrichs and the Godunov schemes are typical examples of E-schemes. It turns out (see [21], p. 482) that the E-schemes are exactly those having no less numerical viscosity than the Godunov scheme. In particular, the Lax-Friedrichs scheme has higher numerical viscosity than the Godunov scheme.

Godunov type schemes for nonlocal equations have been considered in $[7,14]$. In order to define a Godunov scheme for the nonlocal Burgers' equation we first define the convolution term

$$
V_{j+1 / 2}^{n}=\sum_{k=-\ell}^{\ell-1} \gamma_{k} \rho_{j-k+1}^{n}, \quad \text { with } \gamma_{k} \text { as in (2.5). }
$$

By plugging the formula $f(\rho)=V_{j+1 / 2}^{n} \rho$ into (2.7) and recalling (2.6) we arrive at

$$
F_{j+1 / 2}^{n}= \begin{cases}V_{j+1 / 2}^{n} \rho_{j}^{n} & V_{j+1 / 2}^{n} \geq 0 \\ V_{j+1 / 2}^{n} \rho_{j+1}^{n} & V_{j+1 / 2}^{n}<0 .\end{cases}
$$

By plugging the above numerical flux function into (2.2) we obtain a Godunov type scheme for the nonlocal Burgers' equation (1.7). Note that our scheme is slightly different from the one in $[7,14]$ because in $[7,14]$ the authors focus on the case where $\bar{\rho} \geq 0$, which implies that $\rho_{j}^{n} \geq 0$ for every $n$ and $j$ and hence that $V_{j+1 / 2}^{n} \geq 0$. This in turn implies that only the first case at the right hand side of (2.9) can occur. In the present work we consider cases where $\bar{\rho}$ attains negative values (see Example A in Sect. 3.1) and hence we use (2.9).

We conclude this section by discussing a property of the Godunov scheme (2.9) that we need in the following. 
Lemma 2.1. Assume that

$$
\bar{\rho}(x)=0, \quad \text { for every } x>0
$$

and that

$$
\eta_{\varepsilon}(x)=0, \quad \text { for every } x>0 .
$$

Let $\rho_{j}^{n}$ the value of the approximate solution provided by the Godunov scheme (2.2), (2.9). We have

$$
\rho_{j}^{n}=0, \quad \text { for every } n \in \mathbb{N}, j \geq 1 .
$$

Proof. We argue by induction on $n$. By combining (2.1) with (2.10) we conclude that the equality in (2.12) holds true for $n=0$. We now assume that the equality in (2.12) is satisfied by $\rho_{j}^{n}$ and show that it is satisfied by $\rho_{j}^{n+1}$. We combine (2.2) with (2.9) and we point out that $F_{j+1 / 2}^{n}=0$ for every $j \geq 1$ owing to (2.12). We conclude that, to show that the equality in (2.12) is satisfied at $n+1$, it suffices to show that

$$
V_{j-1 / 2}^{n}=0, \quad \text { for every } j \geq 1 .
$$

To establish (2.13), we recall (2.8) and, by combining (2.5) and (2.11), we conclude that $\gamma_{k}=0$ for every $k \geq 0$. By plugging this equality into $(2.8)$ we get

$$
V_{j-1 / 2}^{n} \stackrel{(2.8)}{=} \sum_{k=-\ell}^{\ell-1} \gamma_{k} \rho_{j-k}^{n}=\sum_{k=-\ell}^{-1} \gamma_{k} \rho_{j-k}^{n} \stackrel{(2.12)}{=} 0, \quad \text { if } j \geq 0 .
$$

This yields (2.13) and hence concludes the proof of Lemma 2.1.

\section{Analytical Results}

In this paragraph we briefly discuss the main analytical properties of the examples we will use in our numerical tests.

\subsection{Example A: odd initial datum, isotropic convolution kernels}

Assume that

$$
\rho(0, x)=\bar{\rho}^{(A)}(x):=(x+2) \mathbb{1}_{[-2,-1]}(x)+\mathbb{1}_{[-1,0]}(x)-\mathbb{1}_{[0,1]}(x)+(x-2) \mathbb{1}_{[1,2]}(x),
$$

where here and in the following $\mathbb{1}_{E}$ denotes the characteristic function of the set $E$. The entropy admissible solution of the Cauchy problem for the (local) Burgers' equation (1.6) is

$$
\rho^{(A)}(t, x)= \begin{cases}\frac{x+2}{2 t+1}, & x \in[-2,2 t-1], t \leq \frac{1}{2}, \quad \text { or } x \in[-2,0], t>\frac{1}{2}, \\ 1, & x \in[2 t-1,0], \quad t \leq \frac{1}{2} \\ -1, & x \in[0,1-2 t], \quad t \leq \frac{1}{2} \\ \frac{x-2}{2 t+1}, & x \in[1-2 t, 2], \quad t \leq \frac{1}{2}, \quad \text { or } x \in[0,2], \quad t>\frac{1}{2}, \\ 0, & \text { elsewhere. }\end{cases}
$$

We now consider the Cauchy problem obtained by coupling (3.1) with the nonlocal Burgers' equation (1.7) and we term $\rho_{\varepsilon}^{(A)}$ its solution. We assume furthermore that the convolution kernel $\eta$ is even, i.e. $\eta_{\varepsilon}(x)=\eta_{\varepsilon}(-x)$ for every $x$. The analysis in Section 5.1 of [11] states that, under these assumptions, the family $\rho_{\varepsilon}^{(A)}$ does 
not converge to the entropy admissible solution (3.2) as $\varepsilon \rightarrow 0^{+}$, not even weakly or up to subsequences. We refer to [11] for the precise statements and the technical proof, but loosely speaking the very basic idea is the following. By using the fact that the initial datum $\bar{\rho}^{(A)}$ is odd and that the convolution kernel is even, one can show that the solution of the nonlocal equation is odd and this in turn implies, after some more work, that

$$
\int_{-\infty}^{0} \rho_{\varepsilon}^{(A)}(t, y) \mathrm{d} y=\int_{-\infty}^{0} \bar{\rho}^{(A)}(y) \mathrm{d} y, \quad \text { for every } t>0, \varepsilon>0
$$

On the other hand, the entropy admissible solution of the Burgers' equation satisfies

$$
\int_{-\infty}^{0} \rho^{(A)}(t, y) \mathrm{d} y<\int_{-\infty}^{0} \bar{\rho}^{(A)}(y) \mathrm{d} y, \quad \text { for every } t>0
$$

and by comparing (3.3) and (3.4) and performing some more work one eventually manages to rule out convergence.

\subsection{Example B: positive initial datum, anisotropic convolution kernels}

If

$$
\rho(0, x)=\bar{\rho}^{(B)}(x):=\mathbb{1}_{[-1,0]}(x),
$$

then the entropy admissible solution of the Cauchy problem for the (local) Burgers' equation (1.6) is

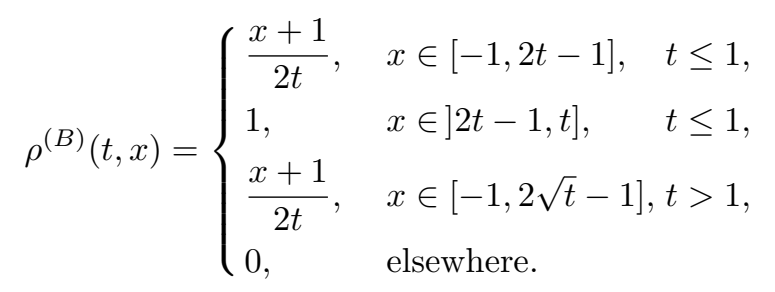

We term $\rho_{\varepsilon}^{(B)}(t, x)$ the solution of the Cauchy problem obtained by coupling (1.7) with (3.5). Assume that the convolution kernels $\eta_{\varepsilon}$ are anisotropic, more precisely they are supported on the negative real line, i.e.

$$
\eta_{\varepsilon}(x)=0, \quad \text { for every } x>0 .
$$

In this case the analysis in Section 5.2 of [11] states that the family $\rho_{\varepsilon}^{(B)}$ does not converge to the entropy admissible solution $(3.10)$ as $\varepsilon \rightarrow 0^{+}$, not even weakly or up to subsequences. The basic reason why $\rho_{\varepsilon}^{(B)}$ does not converge to $\rho^{(B)}$ is because one can show that

$$
\rho_{\varepsilon}^{(B)}(t, x)=0, \quad \text { for every } x>0 \text { and } t>0,
$$

see Lemma 5.3 of [11]. Since $\rho^{(B)}$ does not share this property, then with some more work one manages to rule out convergence.

\subsection{Example C: positive initial datum, isotropic convolution kernels}

If

$$
\rho(0, x)=\bar{\rho}^{(C)}(x):=\mathbb{1}_{[-1,1]}(x),
$$

then the entropy admissible solution of the Cauchy problem for the (local) Burgers' equation (1.6) is

$$
\rho^{(C)}(t, x)= \begin{cases}\frac{x+1}{2 t}, & x \in[-1,2 t-1], \quad t \leq 2 \\ 1, & x \in[2 t-1, t+1], \quad t \leq 2 \\ \frac{x+1}{2 t}, & x \in[-1,2 \sqrt{2 t}-1], t>2 \\ 0, & \text { elsewhere. }\end{cases}
$$


As before, we term $\rho_{\varepsilon}^{(C)}(t, x)$ the solution of the Cauchy problem obtained by coupling (1.7) with (3.9). Assume that the convolution kernels are even functions, i.e. $\eta_{\varepsilon}(x)=\eta_{\varepsilon}(-x)$, for every $x \in \mathbb{R}$. In this case, for every $p>1$ the analysis in Section 5.2 of [11] states that, as $\varepsilon \rightarrow 0^{+}, \rho_{\varepsilon}^{(C)}$ does not converge to $\rho^{(C)}$ strongly in $L^{p}$, not even up to subsequences. Loosely speaking, this is due to the fact that we can single out an entropy that is conserved by $\rho_{\varepsilon}^{(C)}$ and is dissipated by $\rho^{(C)}$.

\subsection{Example D: explicit solution of the nonlocal equations}

If

$$
\rho(0, x)=\bar{\rho}^{(D)}(x):=\mathbb{1}_{]-\infty, 0]}(x),
$$

then the entropy admissible solution of the Cauchy problem for the (local) Burgers' equation (1.6) is the shock

$$
\rho^{(D)}(t, x)= \begin{cases}1 & x \in]-\infty, t] \\ 0 & x \in[t,+\infty[.\end{cases}
$$

Also, consider the nonlocal Burgers' equation (1.7) and assume that the convolution kernel is supported on the positive real axis, i.e. $\eta_{\varepsilon}(x)=0$ for every $x<0$. In this case one can show that, for every $\varepsilon>0$, the solution of the Cauchy problem obtained by coupling (1.7) with (3.11) is exactly the same shock as in (3.12), i.e. $\rho_{\varepsilon}^{(D)} \equiv \rho^{(D)}$.

\subsection{Example E: isotropic convolution kernels, regular limit solution}

Assume that

$$
\rho(0, x)=\bar{\rho}^{(E)}(x):=\frac{1}{4}\left(1+\sin \left(\frac{\pi x}{2}+\frac{\pi}{2}\right)\right) \mathbb{1}_{[-2,0]}(x)+\frac{1}{2} \mathbb{1}_{[0, \infty[}(x) .
$$

Since the initial datum is regular and monotone nondecreasing, classical results on scalar conservation laws rule out shock formation and imply that the solution of the Cauchy problem for the (local) Burgers' equation (1.6) is regular. Consider the nonlocal Burgers' equation (1.7) and assume that the convolution kernels are even, i.e. that $\eta_{\varepsilon}(x)=\eta_{\varepsilon}(-x)$ for every $x \in \mathbb{R}$ and $\varepsilon>0$. Owing to the analytical convergence result by Zumbrun ([22], Prop. 4.1), in this case we expect that the solutions of the nonlocal equation uniformly converge to the solution of the (local) Burgers' equation.

\section{Benchmark numerical test For the LOCAL AND THE NONLOCAL Burgers' EQUATION: TEST 1 (EXAMPle D)}

In this paragraph we discuss some benchmark tests we use to validate our numerical schemes. We do not report for brevity the convergence for both schemes in the case of the local Burgers equation, which is a wellknown result. Instead, Test 1 is designed to validate the numerical schemes for the nonlocal equation. We take the same initial datum $\bar{\rho}^{(D)}$ as in (3.11) and the convolution kernel

$$
\eta_{\varepsilon}(x):=\alpha_{\varepsilon}(|x-\varepsilon||x|)^{5 / 2} \mathbb{1}_{[0, \varepsilon]}(x),
$$

where (here and in the following) the constant $\alpha_{\varepsilon}>0$ is chosen in such a way that $\eta_{\varepsilon}$ has unit integral. The exact value of $\alpha_{\varepsilon}$ can vary from occurrence to occurrence. As pointed out in Section 3.4, in this case the solution of the Cauchy problem for the nonlocal Burgers' equation (1.7) is explicit and it is given by (3.12). We can then validate the schemes for the nonlocal equation by computing the $L^{1}$ norm in space of the difference between the numerical solution (obtained with the Lax-Friedrichs and the Godunov type schemes) and the exact analytical solution. The results are displayed in Figure 1. We evaluate the $L^{1}$ norm at time $t=2$ for different values of the nonlocal parameter $\varepsilon$, and we evaluate the convergence with respect to mesh size $h$. The results show convergence of order $1 / 4$ in average (computed by means of linear regression) of the numerical solution to the analytical solution for the nonlocal equation. 


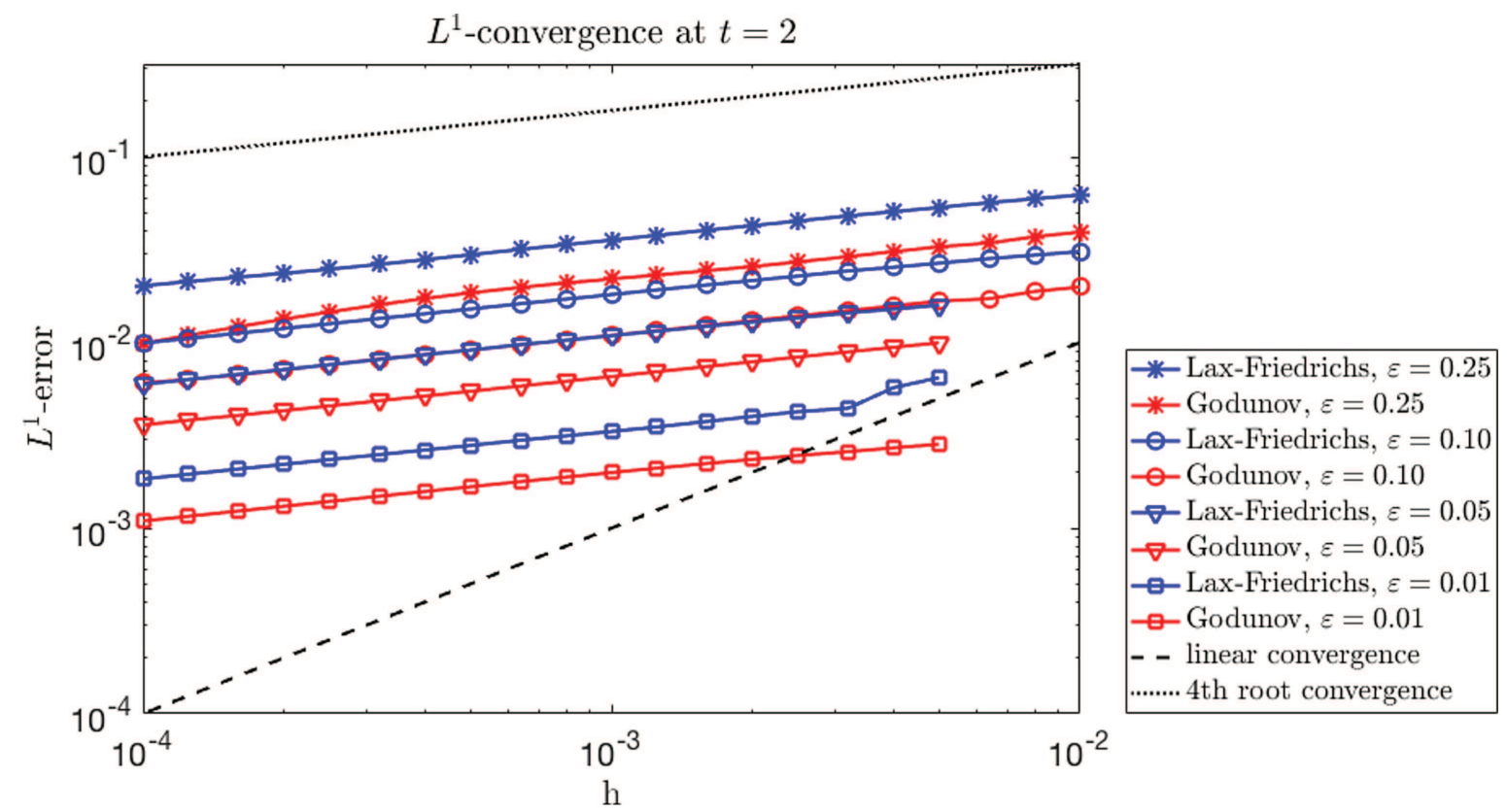

Figure 1. Test 1 (Example D), $L^{1}$-Convergence of the numerical schemes, Lax-Friedrichs and Godunov, with respect to the mesh size $h$, for the nonlocal equation at $\varepsilon=0.25,0.1,0.05,0.01$ and $t=2$.

\section{NumericAl TESTS ON THE NONLOCAL-TO-LOCAL LIMIT}

We now discuss some numerical tests that aim at investigating the nonlocal-to-local limit from (1.2) to (1.3). Before entering into the details of each test, we make a preliminary remark. Our main goal in the present paper is to investigate whether or not standard numerical schemes (the Lax-Friedrichs and the Godunov schemes discussed in Sect. 2) are suited to numerically study the nonlocal-to-local limit from (1.2) to (1.3). We do this by keeping the mesh size $h$ fixed in several numerical experiments and letting the nonlocal parameter $\varepsilon$ vary in the range $\varepsilon>h$ (see the comment after formula (2.5)). As a byproduct, this choice allows us to obtain a direct comparison with the numerical experiments concerning the nonlocal-to-local limit in Section 3.3 of [2], because in those experiments the mesh size is fixed. Additionally, we also discuss numerical results where we simultaneously vary the mesh size $h$ and the nonlocal parameter $\varepsilon$.

\subsection{Test 2 (Example E): isotropic convolution kernels and regular limit solution}

In Test 2 we consider the same monotone increasing initial datum $\bar{\rho}^{(E)}$ as in (3.13) and the isotropic convolution kernels

$$
\eta_{\varepsilon}(x)=\alpha_{\varepsilon}(|x-\varepsilon||x+\varepsilon|)^{5 / 2} \mathbb{1}_{[-\varepsilon, \varepsilon]}(x) .
$$

As pointed out in Section 3.5, owing to a result by Zumbrun ([22], Prop. 4.1) from the analytical viewpoint we expect that in this case the solutions of the nonlocal equations converge to the (regular) solution of the Burgers' equation.

In Test 2 we compute the numerical solutions of the nonlocal equations with the Lax-Friedrichs type and the Godunov type methods and we compare it with the entropy admissible solution of the (local) Burgers' equation, computed with the Lax-Friedrichs and the Godunov scheme, respectively. We display the results in Figure 2: the results obtained with both the Lax-Friedrichs and the Godunov type schemes suggest convergence. In the case of Test 2 this agrees with the analytical results. 


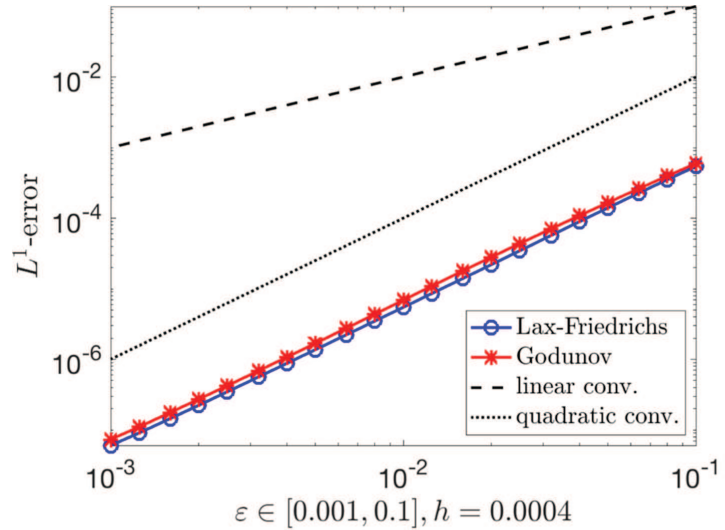

(a)

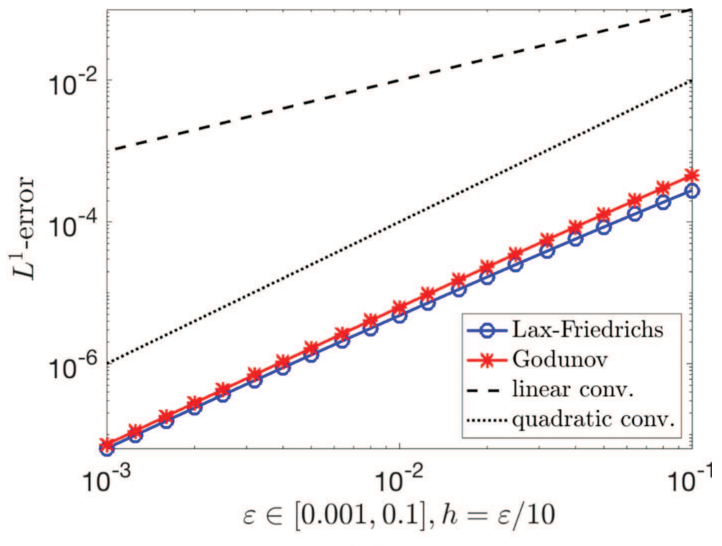

(b)

Figure 2. Test 2 (Example E), $L^{1}$-error at $t=2$, for different values of $\varepsilon$, comparing the solutions of the nonlocal equations with the entropy solution of the (local) Burgers' equation for both numerical schemes, when (a) $h=0.0004$, (b) $h=\varepsilon / 10$.

\subsection{Test 3 (Example A): odd initial datum, isotropic convolution kernels}

In Test 3 we take the same initial datum $\bar{\rho}^{(A)}$ as in (3.1) and the same isotropic convolution kernels as in (5.1). As pointed out in Section 3.1, in this case the analysis in [11] implies that the solutions of the nonlocal equation (1.7) do not converge to the entropy admissible solution of the Burgers' equation, which is given by $(3.2)$.

In Test 3 we compute the numerical solution of the nonlocal equation by using the Lax-Friedrichs and the Godunov type schemes. Several snapshots of the solution are displayed in Figure 3. Also, we compare the numerical solution of the nonlocal equation computed with the Lax-Friedrichs and the Godunov scheme with the entropy admissible solution of the (local) Burgers' equation, computed with the Lax-Friedrichs and the Godunov scheme, respectively. More precisely, we evaluate the $L^{1}$ norm of the difference at time $t=2$ and for different values of the convolution parameter $\varepsilon$. We show the corresponding results in Figure 4.

Here are the main remarks concerning the numerical results for Test 3.

(i) Figure 4, part (a) shows the numerical results obtained by keeping the space mesh fixed and varying the convolution parameter $\varepsilon$. The numerical results for both schemes strongly suggest that the $L^{1}$ norm of the difference converges to 0 when $\varepsilon \rightarrow 0^{+}$. As pointed out in Section 3.1, in this case we can analytically rule out convergence in the nonlocal-to-local limit and hence the numerical evidence provides the wrong intuition. Owing to the discussion in Section 1.2, this is most likely due to the presence in both schemes of the numerical viscosity.

(ii) In Figure 4, part (b), we display the results obtained by simultaneously varying the space mesh $h$ and the convolution parameter $\varepsilon$. More precisely, we choose $h$ of the order of $\varepsilon^{2}$ : in this way, the space mesh goes to 0 much faster than the convolution parameter. The results in part (b) are qualitatively similar to the results in part (a) for the Lax-Friedrichs scheme, whereas the curve for the Godunov scheme is much flatter, which does not support convergence in the nonlocal-to-local limit. This is more in accordance with the analytical results, which rule out convergence for this example.

Wrapping up, Test 3 shows that the numerical viscosity may jeopardize the reliability of the numerical investigation of the nonlocal-to-local limit. 
Analytical solution of the (local) Burgers' equation
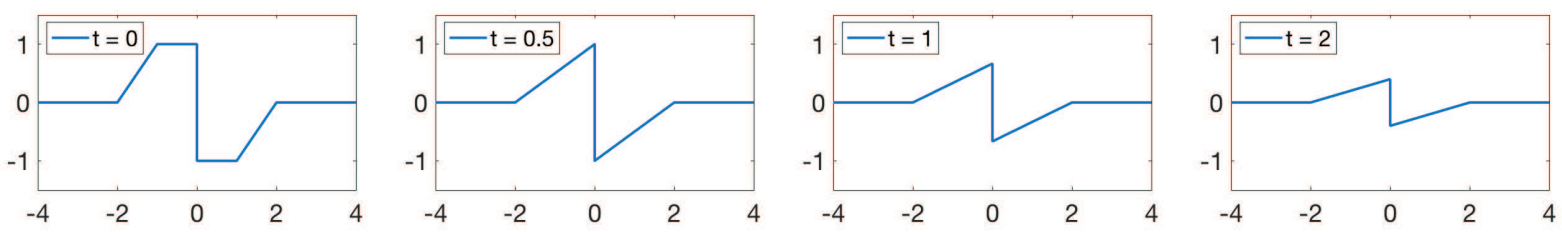

Numerical solution of the (local) Burgers' equation computed with the Lax-Friedrichs method
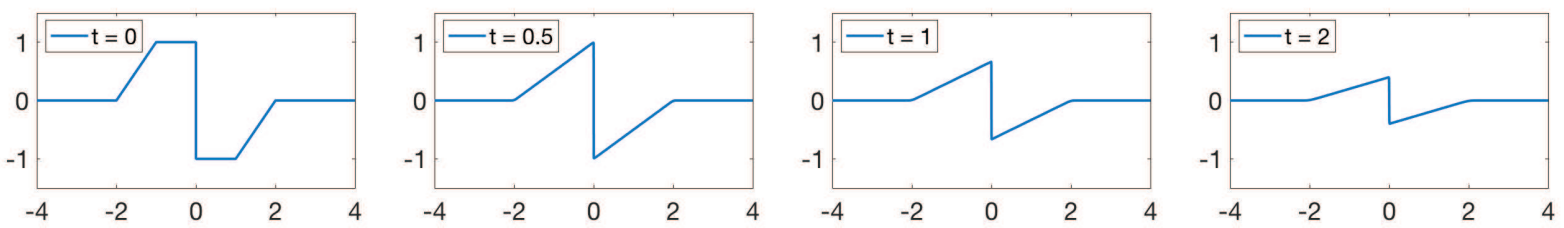

Numerical solution of the (local) Burgers' equation computed with the Godunov method
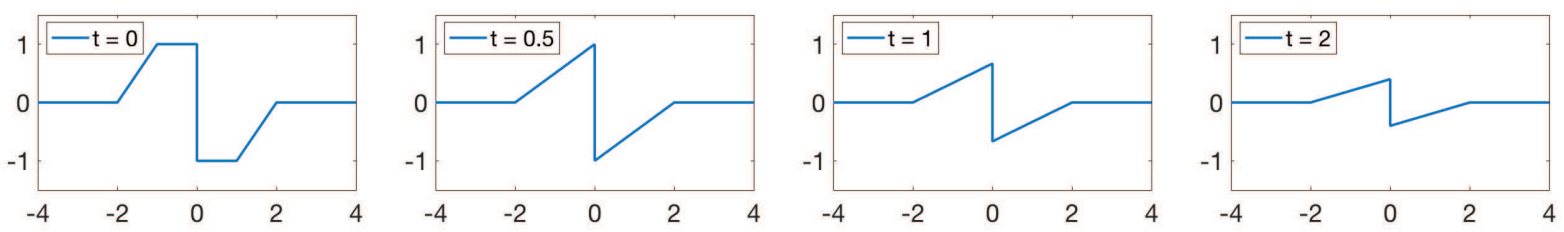

Numerical solution of the nonlocal Burgers' equation computed with the Lax-Friedrichs type method
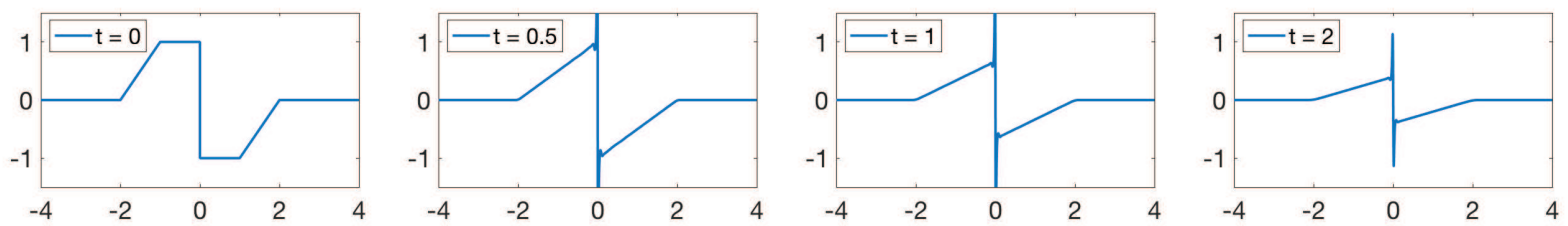

Numerical solution of the nonlocal Burgers' equation computed with the Godunov type method
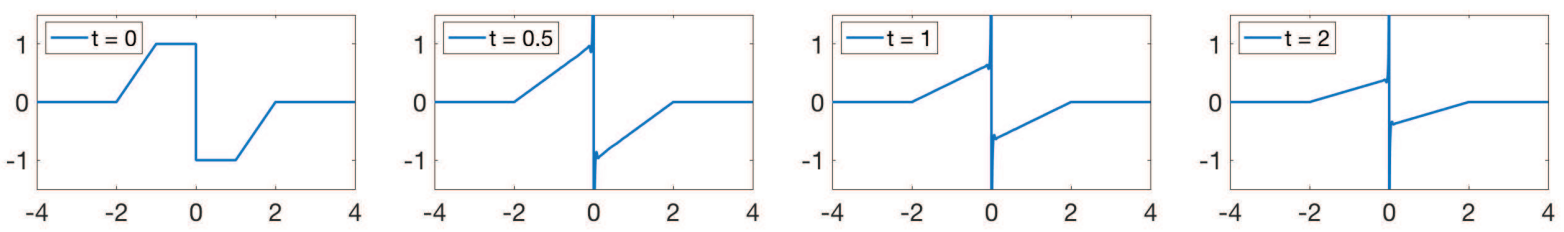

Figure 3. Test 3 (Example A), snapshots of solution of Burgers' equation with initial condition (3.1) and isotropic convolution kernel, when $\varepsilon=0.1, h=0.0004$.

\subsection{Test 4 (Example B): anisotropic convolution kernel, positive initial datum}

The goal of Test 4 is twofold: first, it again shows that the Lax-Friedrichs scheme can erroneously suggest that the solutions of the nonlocal equation converge to the entropy admissible solution of the conservation law as the nonlocal parameter $\varepsilon \rightarrow 0^{+}$in cases where this nonlocal-to-local convergence is ruled out by analytical results (see Fig. 5). The numerical results obtained by the Godunov scheme do not suggest convergence and are therefore more in accordance with the analytical results. The second goal of Test 4 is to show that the 


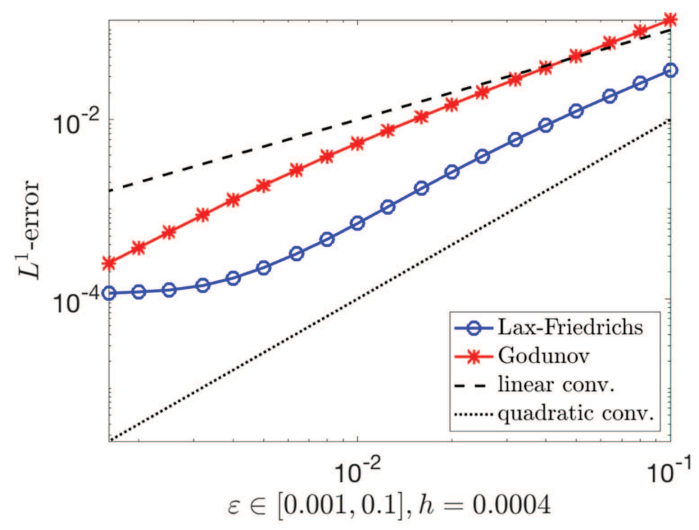

(a)

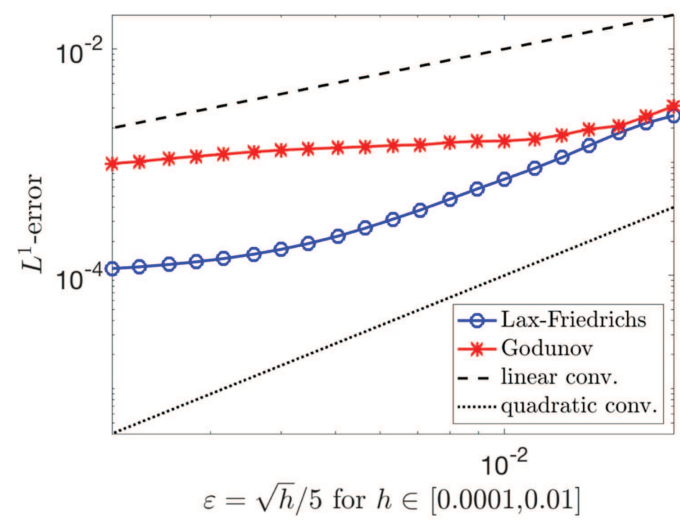

(b)

Figure 4. Test 3 (Example A), $L^{1}$-error at $t=2$, for different values of $\varepsilon$, comparing the nonlocal solution to the local solution for both numerical schemes: (a) fixed viscosity $h=0.0004$, (b) varying viscosity $h=25 \varepsilon^{2}$.

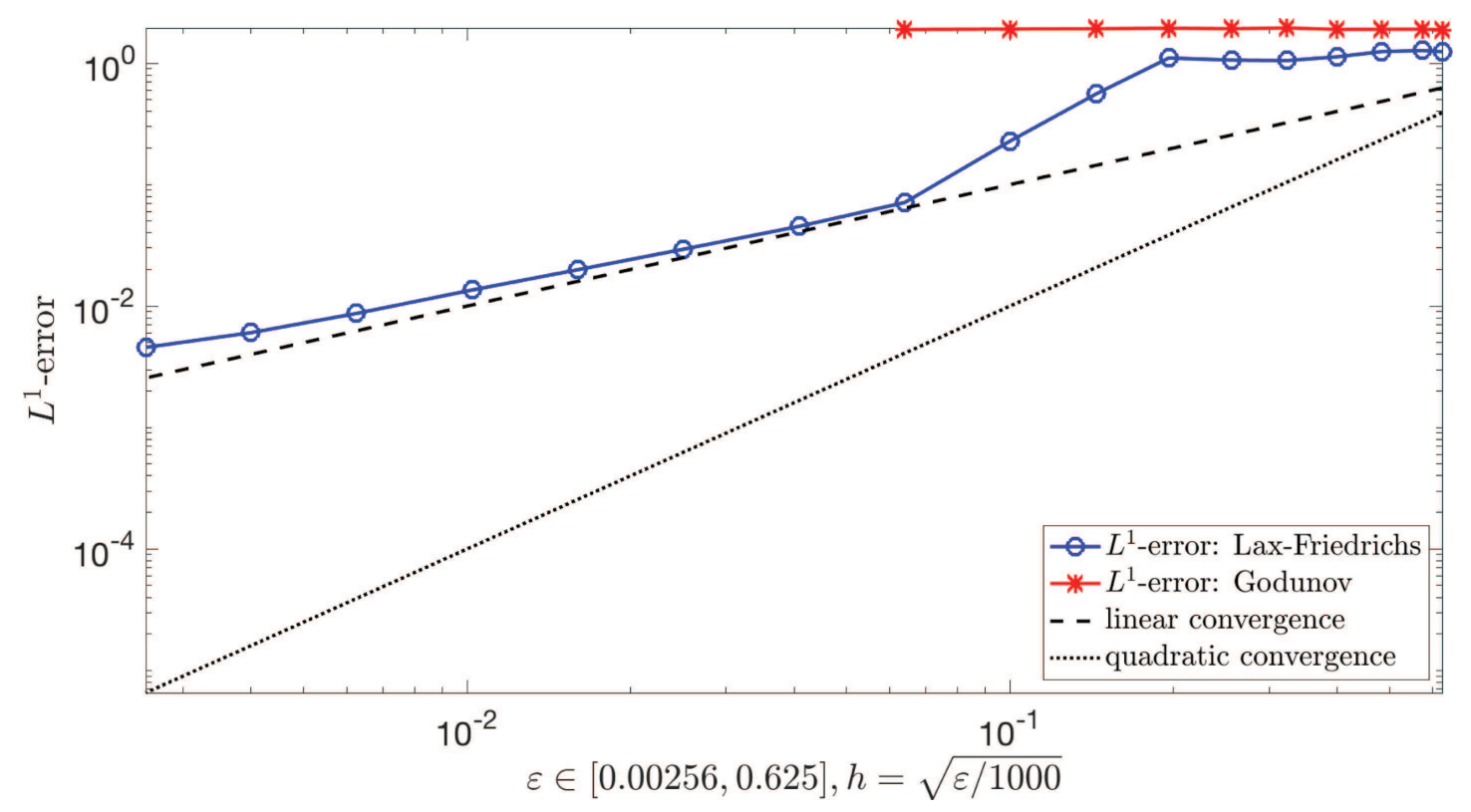

Figure 5 . Test 4 (Example B), $L^{1}$-error at $t=2$, for different values of $\varepsilon$, comparing the solutions of the nonlocal equations with the entropy solution of the local equation for both numerical schemes and for varying viscosity $h$ such that $\varepsilon=1000 h^{2}$. The $L^{1}$ error of the Godunov scheme is much higher for small values of $\varepsilon$. 
Analytical solution
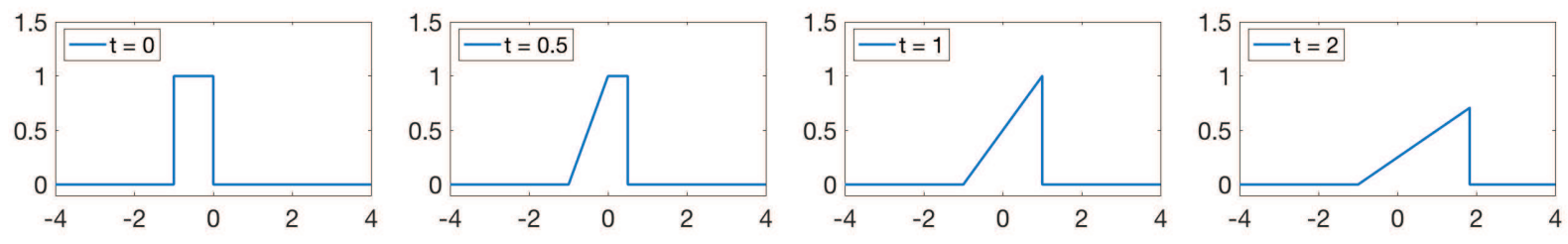

Numerical solution of the (local) Burgers' equation with Lax-Friedrichs method
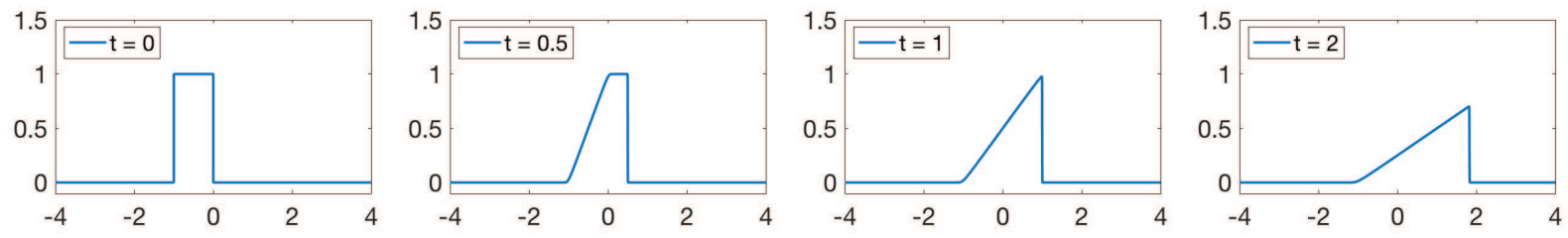

Numerical solution of the (local) Burgers' equation with Godunov method
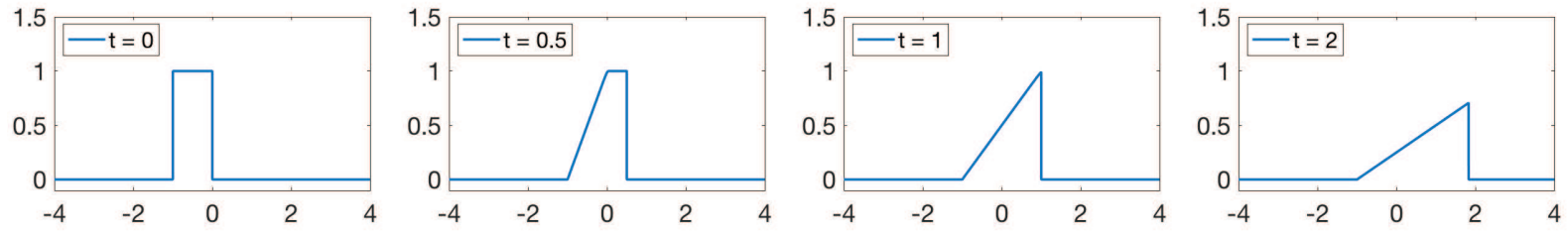

Numerical solution of the nonlocal Burgers' equation with Lax-Friedrichs method
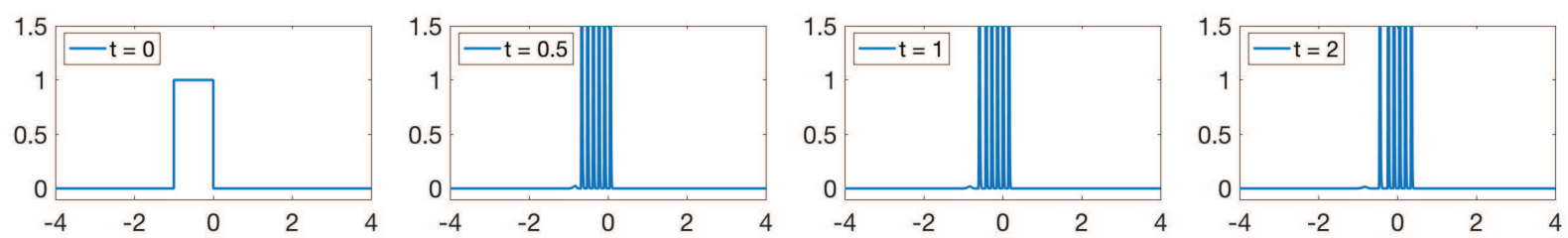

Numerical solution of the nonlocal Burgers' equation with Godunov method
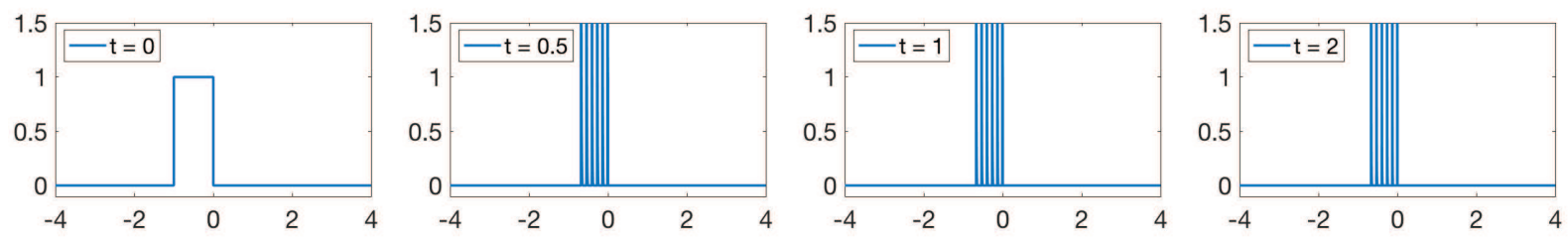

Figure 6. Test 4 (Example B), snapshots of the solution of Burgers' equation with initial condition and convolution kernel (3.5), when $\varepsilon=0.1, h=0.0004$.

Lax-Friedrichs schemes may fail to capture relevant qualitative properties of the analytical solution of the nonlocal equations. Indeed, in the case of Test 4 the analytical solution of the nonlocal equations are supported on the negative real axis: the numerical solutions computed with the Lax-Friedrichs scheme do not satisfy this property, whereas owing to Lemma 2.1 the Godunov scheme preserves this property. We now provide the technical details concerning Test 4: we take the same initial datum $\bar{\rho}^{(B)}$ as in (3.5) and the convolution kernels

$$
\eta_{\varepsilon}(x)=\alpha_{\varepsilon}(|x||x+\varepsilon|)^{5 / 2} \mathbb{1}_{[-\varepsilon, 0]}(x) .
$$


Note that these convolution kernels satisfy (3.7) and hence the discussion in Section 3.2 applies. In particular, the analytical solutions $\rho_{\varepsilon}^{(B)}$ of the nonlocal equations (1.7) are all supported on the negative axis, i.e. satisfy (3.8), and do not converge to the solution of the (local) Burgers' equation.

In Test 4 we compute the numerical solution of the nonlocal equations (1.7) by using the Lax-Friedrichs type method and the Godunov type method and we display the corresponding results in Figures 5 and 6 . More precisely, Figure 5 displays the behavior of the $L^{1}$ norm of the difference between the solutions of the nonlocal equations, computed with the Lax-Friedrichs and the Godunov scheme, and the entropy admissible solution of the (local) Burgers' equation at time $t=2$, computed with the Lax-Friedrichs and the Godunov scheme, respectively. Recall that the analytical solution is given by (3.6). Figure 6 shows the snapshots of the solution at different times. We now comment on Figures 5 and 6.

(i) The results in Figure 5 obtained with the Lax-Friedrichs type method suggest that the solutions of the nonlocal equation converge to the entropy admissible solution of the Burgers' equation. This contradicts the analytical results discussed in Section 3.2. On the other hand, the numerical results obtained with the Godunov type scheme do not suggest convergence and hence are more in accordance with the analytical results in Section 3.2. This is most likely due to the fact that the Lax-Friedrichs type scheme has higher numerical viscosity and hence it is not reliable to test the nonlocal-to-local limit. The Godunov type scheme has less numerical viscosity and is, at least in this case, more reliable.

(ii) The snapshots of the solution obtained with the Godunov type and the Lax-Friedrichs type schemes show that the Godunov scheme is, at least in this case, better at preserving analytical properties of the solution of the nonlocal equation. Indeed, the exact solution of the nonlocal Burgers' equation is supported on the negative axis, i.e. satisfies (3.8). Owing to Lemma $2.1^{2}$, this important analytical property is satisfied by the numerical solution obtained by the Godunov type method, and this is also confirmed by the snapshots in Figure 6. On the other hand, the snapshots on the forth row of Figure 6 show that property (3.8) is not satisfied by the solutions obtained by the Lax-Friedrichs type method.

The take-home message from Test 4 is the following: the Godunov type scheme is in this case more reliable than the Lax-Friedrichs type scheme for the numerical investigation of the nonlocal-to-local limit. The Godunov scheme is, at least in this case, also better at preserving relevant qualitative properties of the solution.

\subsection{Test 5 (Example C): positive density and isotropic convolution kernels}

In Test 5 we take the same initial datum as in (3.9) and the same convolution kernels as in (5.1). Since the convolution kernels are even functions, we can apply the discussion in Section 3.3 and conclude that, for every $p>1$, the solutions of the nonlocal equations do not converge in the nonlocal-to-local limit to the entropy admissible solution of the (local) Burgers' equation strongly in $L^{p}$. In Test 5 we compute the numerical solution of the nonlocal equations with the Lax-Friedrichs type and with the Godunov type methods. We display the corresponding results in Figure 7 (convergence analysis) and in Figure 8 (snapshots of the solution). More precisely, Figure 7 shows the $L^{p}$ norm of the difference computed at time $t=2$ between the numerical solutions of the nonlocal equations computed with the Lax-Friedrichs and the Godunov method and the entropy admissible solution of the (local) Burgers' equation, computed with the Lax-Friedrichs and the Godunov method, respectively. Here are the main comments.

(i) In the results displayed in Figure 7, part (a), we keep the space mesh $h$ fixed and we consider smaller and smaller values of the convolution parameter $\varepsilon$. The $L^{p}$ error is clearly smaller in the case of the LaxFriedrichs scheme with respect to the case of Godunov, for every tested value of $p>1$, and this means that the results obtained with the Godunov scheme are more in accordance with the analytical results, which in this example rule out convergence in the nonlocal-to-local limit.

\footnotetext{
${ }^{2}$ More precisely, Lemma 2.1 states that the numerical solution computed with the Godunov scheme is supported on ] $\left.-\infty, h / 2\right]$, where $h$ is the mesh size.
} 


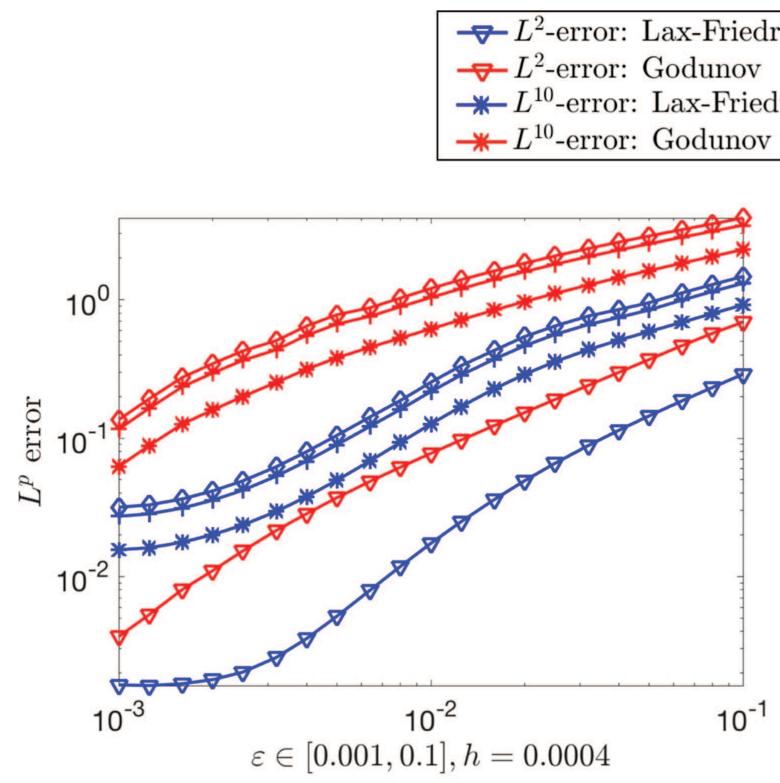

(a)
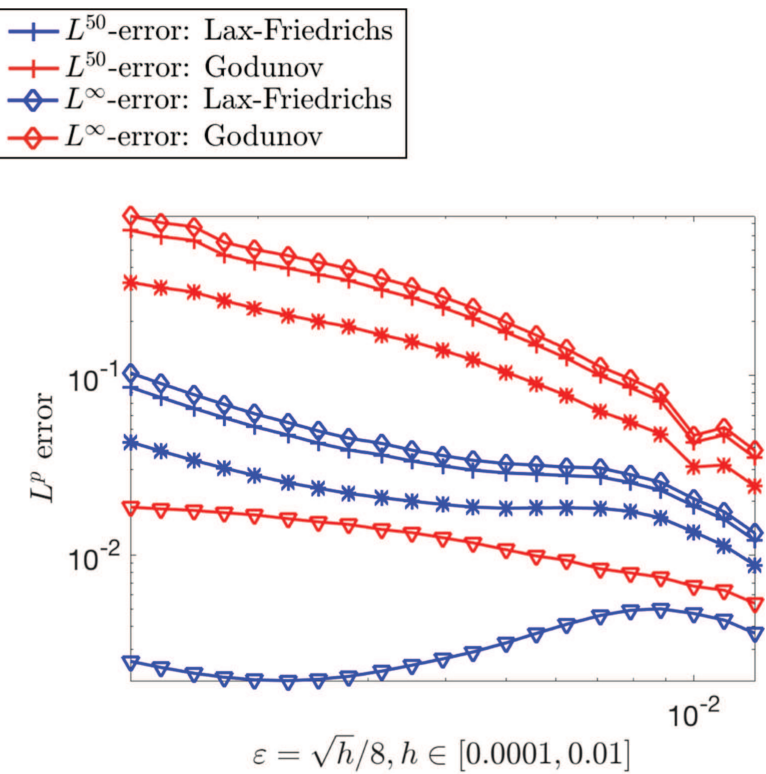

(b)

Figure 7 . Test 5 (Example C), $L^{p}$-error at $t=2, p \geq 1$, at different values of $\varepsilon$, comparing the nonlocal solution to the local solution for both numerical schemes: (a) fixed viscosity $h=0.0004$, (b) varying viscosity $h=64 \varepsilon^{2}$.

(ii) Figure 7, part (b) displays numerical results where the space mesh $h$ is of the order of $\varepsilon^{2}$. In this case the numerical results obtained with both schemes do not suggest convergence in $L^{p}$ for $p>1$ and hence agree with the analytical results. This is most likely due to the fact that the numerical viscosity goes to 0 very fast and hence does not affect the investigation of the nonlocal-to-local limit. Also in this case, for fixed $p$ the $L^{p}$-error computed for the Godunov solution is always bigger than the corresponding $L^{p}$-error computed for the Lax-Friedrichs solution, which means that the results obtained with the Godunov scheme are more in accordance with the analytical results.

In a nutshell, Test 5 shows that the presence of the numerical viscosity can jeopardize the reliability of the numerical schemes. In Test 5 , the most reliable results are obtained by taking the scheme with the smallest numerical viscosity.

\section{Conclusion}

In the present paper we argue that the numerical investigation of the nonlocal-to-local limit for nonlocal conservation laws is a fairly subtle problem. We have shown that particular attention must be paid to the numerical viscosity which can, for some values of the mesh size and of the parameter $\varepsilon$, jeopardize the reliability of the numerical experiments.

This claim is supported by the following instances:

- Lax-Friedrichs type schemes have high numerical viscosity and erroneously suggest convergence in cases where convergence is ruled out by analytical considerations (see in particular Test 3). Also, Lax-Friedrichs type scheme fail to capture relevant qualitative properties of the solutions of the nonlocal equations (see in particular Test 4). 

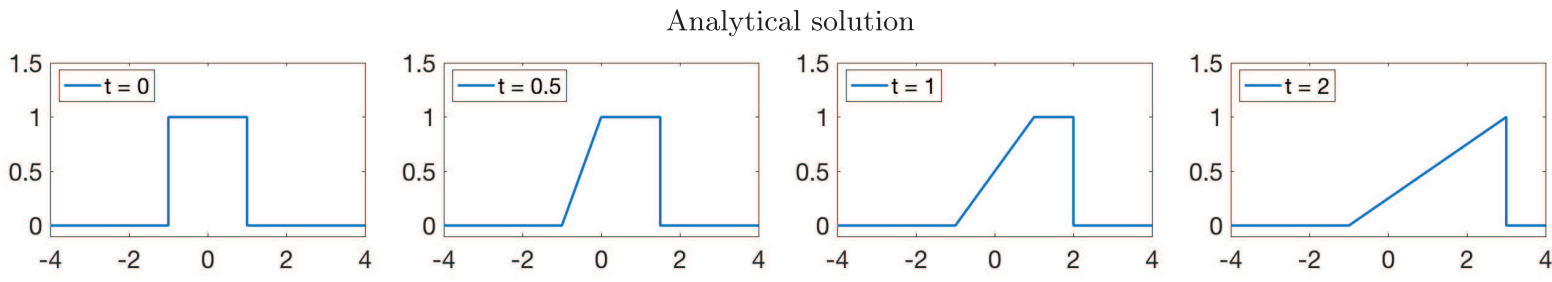

Numerical solution of the inviscid Burgers' equation with Lax-Friedrichs method
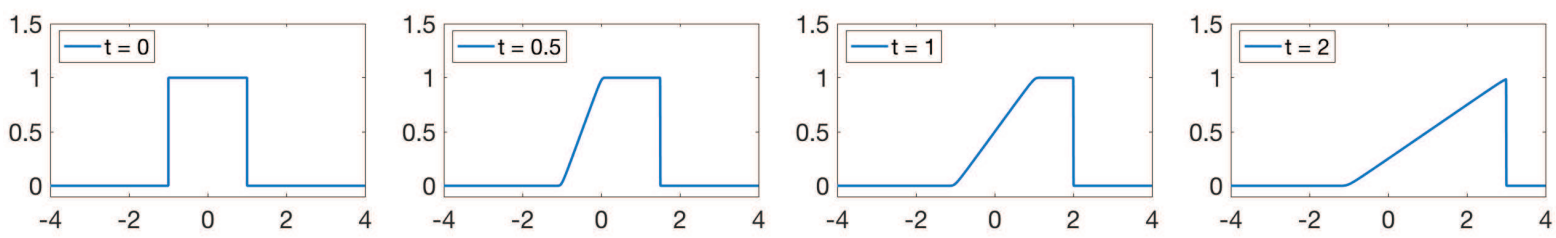

Numerical solution of the inviscid Burgers' equation with Godunov method
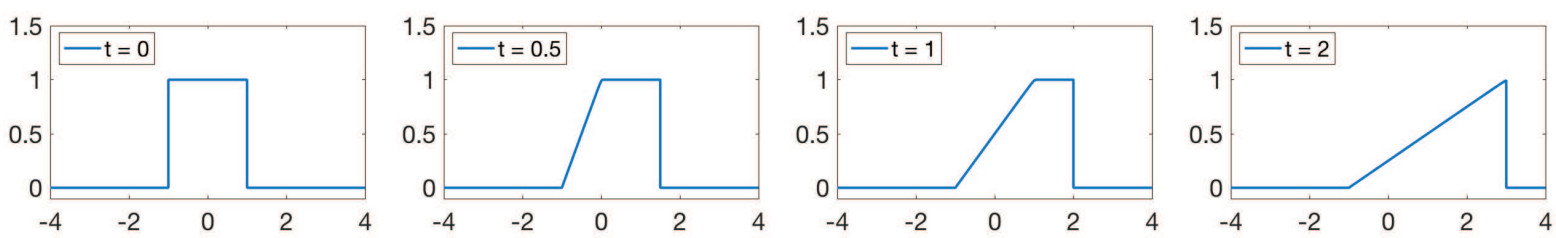

Numerical solution of the nonlocal Burgers' equation with Lax-Friedrichs method
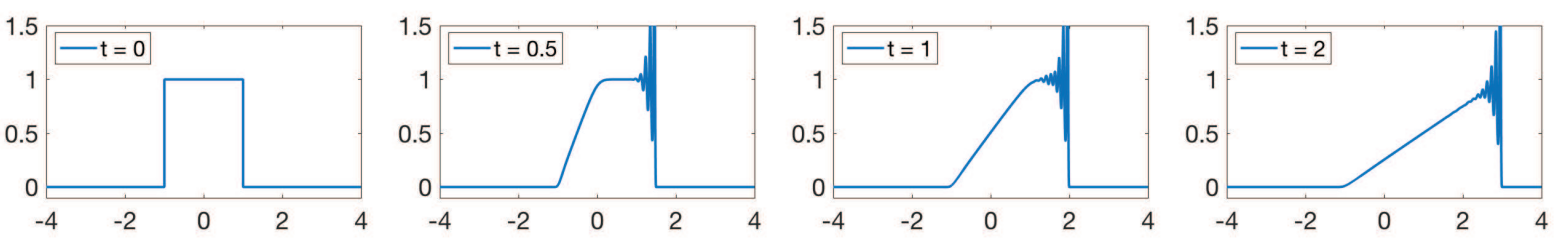

Numerical solution of the nonlocal Burgers' equation with Godunov method
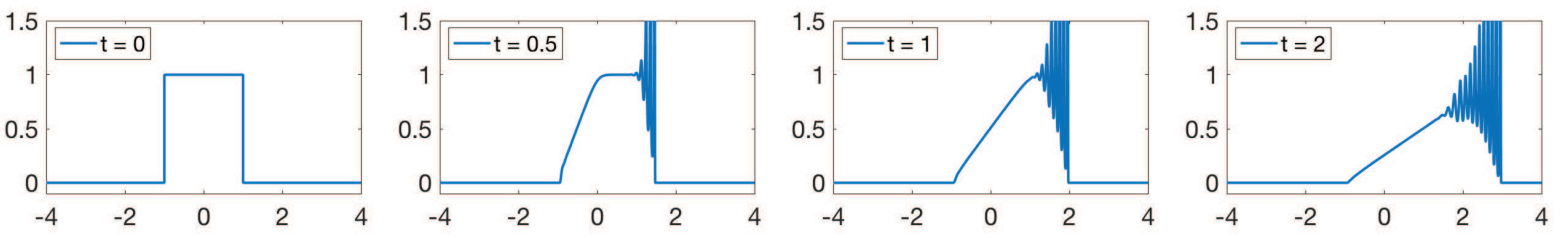

Figure 8. Test 5 (Example C), snapshots of solution of Burgers' equation with initial condition (3.9) and convolution kernel (5.1), when $\varepsilon=0.1, h=0.0004$.

- Godunov type schemes have lower numerical viscosity than Lax-Friedrichs type schemes and, at least in some cases, provide more reliable information on the nonlocal-to-local limit, see Test 4. Also, they appear to be better than Lax-Friedrichs type schemes at capturing relevant qualitative properties of the solutions of the nonlocal equations (see Test 4).

- The numerical results that are most in accordance with the analytical results use the Godunov type scheme with a very low numerical viscosity (i.e. we choose the space mesh of the order $\varepsilon^{2}$, where $\varepsilon$ is the convolution parameter), see Test 5 . 
We feel that the present work could pave the way for several interesting developments. Our work shows that both Lax-Friedrichs and Godunov type schemes, especially on coarse meshes, are not completely reliable to gain analytical intuition on the nonlocal-to-local limit. It would be very interesting, for both numerical and analytical purposes $^{3}$, to introduce numerical schemes providing reliable results for the study of the local limit of nonlocal conservation laws. To this end, a possible future direction is working with Lax-Wendroff type schemes ${ }^{4}$. This is motivated by the fact that the model equation for the Lax-Wendroff equation is a third order equation with no viscous term, see [18]. See also the non-dissipative scheme used in [16].

Acknowledgements. The authors wish to thank Blanca Ayuso de Dios and Giovanni Russo for interesting discussions. MC is partially supported by the Swiss National Science Foundation grant 182565 . GC is partially supported by the Swiss National Science Foundation grant 200021-140232 and by the ERC Starting Grant 676675 FLIRT. LVS is a member of the GNAMPA group of INDAM and of the PRIN National Project "Hyperbolic Systems of Conservation Laws and Fluid Dynamics: Analysis and Applications". MG was partially supported by the Swiss National Science Foundation grant P300P2-167681. Part of this work was done when MC and LVS were visiting the University of Basel: its kind hospitality is gratefully acknowledged.

\section{REFERENCES}

[1] A. Aggarwal, R.M. Colombo and P. Goatin, Nonlocal systems of conservation laws in several space dimensions. SIAM J. Numer. Anal. 53 (2015) 963-983.

[2] P. Amorim, R.M. Colombo and A. Teixeira, On the numerical integration of scalar nonlocal conservation laws. ESAIM: M2AN 49 (2015) 19-37.

[3] F. Betancourt, R. Bürger, K.H. Karlsen and E.M. Tory, On nonlocal conservation laws modelling sedimentation. Nonlinearity 24 (2011) 855-885.

[4] S. Blandin and P. Goatin, Well-posedness of a conservation law with non-local flux arising in traffic flow modeling. Numer. Math. 132 (2016) 217-241.

[5] A. Bressan and W. Shen, On traffic flow with nonlocal flux: a relaxation representation. Arch. Rat. Mech. Anal. 237 (2020) 1213-1236.

[6] A. Bressan and W. Shen, Entropy admissibility of the limit solution for a nonlocal model of traffic flow. Comm. Math. Sci. 19 (2021) 1447-1450.

[7] F.A. Chiarello and P. Goatin, Non-local multi-class traffic flow models. Netw. Heterog. Media 14 (2019) $371-387$.

[8] G.M. Coclite, J.-M. Coron, N. De Nitti, A. Keimer and L. Pflug, A general result on the approximation of local conservation laws by nonlocal conservation laws: the singular limit problem for exponential kernel. Preprint arXiv: 2012.13203 (2020).

[9] G.M. Coclite, N. De Nitti, A. Keimer and L Pflug, Singular limits with vanishing viscosity for nonlocal conservation laws. Nonlinear Anal. Theory Methods App. 211 (2021) 112370.

[10] R.M. Colombo, M. Garavello and M. Lécureux-Mercier, A class of nonlocal models for pedestrian traffic. Math. Models Methods Appl. Sci. 22 (2012) 1150023, 34.

[11] M. Colombo, G. Crippa and L.V. Spinolo, On the singular local limit for conservation laws with nonlocal fluxes. Arch. Rat. Mech. Anal. 233 (2019) 1131-1167.

[12] M. Colombo, G. Crippa, E. Marconi and L.V. Spinolo, Local limit of nonlocal traffic models: convergence results and total variation blow-up. Ann. Inst. Henri Poincaré Anal. Non Linéaire 38 (2021) 1653-1666.

[13] G. Crippa and M. Lécureux-Mercier, Existence and uniqueness of measure solutions for a system of continuity equations with non-local flow. NoDEA Nonlinear Differ. Equ. Appl. 20 (2013) 523-537.

[14] J. Friedrich, O. Kolb and S. Göttlich, A Godunov type scheme for a class of LWR traffic flow models with non-local flux. Netw. Heterog. Media 13 (2018) 531-547.

[15] P. Goatin and S. Scialanga, Well-posedness and finite volume approximations of the LWR traffic flow model with non-local velocity. Netw. Heterog. Media 11 (2016) 107-121.

[16] A. Keimer and L. Pflug, On approximation of local conservation laws by nonlocal conservation laws. J. Math. Anal. App. 475 (2019) 1927-1955.

[17] S.N. Kružkov, First order quasilinear equations with several independent variables. Mat. Sb. (N.S.) 81 (1970) $228-255$.

[18] R.J. LeVeque, Finite Volume Methods for Hyperbolic Problems. Cambridge Texts in Applied Mathematics. Cambridge University Press, Cambridge (2002).

\footnotetext{
${ }^{3}$ Reliable numerical scheme could for instance provide valuable intuition on some analytical open questions. As an example, we mention traffic models with completely anisotropic convolution kernels, which take into account the fact that drivers only look forward, not backward, and hence decide their speed based on the downstream traffic density only. In this case Question 1 on the nonlocal-to-local limit is in its full generality presently open (see however the recent results in $[5,6,8,12,16]$ ).

${ }^{4}$ We thank Giovanni Russo for this remark.
} 
[19] S. Osher, Riemann solvers, the entropy condition, and difference. SIAM J. Numer. Anal. 21 (1984) 217-235.

[20] E. Tadmor, Numerical viscosity and the entropy condition for conservative difference schemes. Math. Comput. 43 (1984) 369-381.

[21] E. Tadmor, Chapter 18 - Entropy stable schemes. In: Handbook of Numerical Methods for Hyperbolic Problems, edited by R. Abgrall and C.-W. Shu. Vol. 17 of Handbook of Numerical Analysis. Elsevier (2016) 467-493.

[22] K. Zumbrun, On a nonlocal dispersive equation modeling particle suspensions. Quart. Appl. Math. 57 (1999) 573-600.

\section{Subscribe to Open (S2O) A fair and sustainable open access model}

This journal is currently published in open access under a Subscribe-to-Open model (S2O). S2O is a transformative model that aims to move subscription journals to open access. Open access is the free, immediate, online availability of research articles combined with the rights to use these articles fully in the digital environment. We are thankful to our subscribers and sponsors for making it possible to publish this journal in open access, free of charge for authors.

\section{Please help to maintain this journal in open access!}

Check that your library subscribes to the journal, or make a personal donation to the S2O programme, by contacting subscribers@edpsciences.org

More information, including a list of sponsors and a financial transparency report, available at: https://www. edpsciences.org/en/maths-s2o-programme 